\title{
The Classical Binary and Triplet Distribution Functions for Dilute Relativistic Plasma
}

\author{
N. A. Hussein'1, D. A. Eisa², E. G. Sayed' \\ ${ }^{1}$ Mathematics Department, Faculty of Science, Assiut University, Assiut, Egypt \\ ${ }^{2}$ Mathematics Department, Faculty of Science, Assiut University, New Valley, Egypt \\ Email: aragamal@yahoo.com
}

Received 1 January 2015; accepted 19 January 2015; published 22 January 2015

Copyright (C) 2015 by authors and Scientific Research Publishing Inc.

This work is licensed under the Creative Commons Attribution International License (CC BY).

http://creativecommons.org/licenses/by/4.0/

C) (i) Open Access

\section{Abstract}

The aim of this paper is to calculate the binary and triplet distribution functions for dilute relativistic plasma in terms of the thermal parameter $\mu$ where $\mu=\frac{m c^{2}}{K T}, m$ is the mass of charge; $c$ is the speed of light; $K$ is the Boltzmann's constant; and $T$ is the absolute temperature. Our calculations are based on the relativistic Bogoliubov-Born-Green-Kirkwood-Yvon (BBGKY) hierarchy. We obtain classical binary and triplet distribution functions for one- and two-component plasmas.

Keywords

Relativistic Plasma, Binary and Triplet Distribution Functions, BBGKY Hierarchy

\section{Introduction}

Studying the properties of plasma has received a great interest in both astrophysical and laboratory plasma application. The relativistic binary distribution function is one of the most important functions of statistical mechanics. The importance of the distribution function in statistical mechanics is due to the fact that all the thermodynamic quantities, such as the pressure, the internal energy and the free energies, can be calculated from it.

Relativistic statistical mechanics has a long story, but we may notice that, whereas the theory of relativistic ideal gases has received deep and detailed developments, little has been achieved in order to account for mutual interactions between particles [1]. In statistical physics, the BBGKY hierarchy (Bogoliubov-Born-GreenKirkwood-Yvon hierarchy, sometimes called Bogoliubov hierarchy) is a set of equations describing the dynam- 
ics of a system of a large number of interacting particles. The equation for an $s$-particle distribution function (probability density function) in the BBGKY hierarchy includes the $(s+1)$-particle distribution function thus forming a coupled chain of equations [2]. Their history of 70 years has brought enormous progress in the investigation of the transition from the microscopic to the macroscopic world, and they are still an attractive starting point for new developments. In particular, great advance has come by clever methods of truncation, approximation and scaling limits of the hierarchy, providing in various cases a justification of the kinetic equations describing particle systems on mesoscopic (intermediate) scales [3]. Many authors studied the BBGKY hierarchy [4] and [5]; Hussein and Eisa [6] [7] calculated the binary and triplet distribution function for one- and twocomponent plasmas for quantum and classical non-relativistic plasma. In this paper, we will calculate these distributions in the relativistic case in terms of plasma thermal parameter. Arendt and Eilek [8] showed that the pair-plasma distribution functions could be described by a thermal parameter $\mu=\frac{m c^{2}}{K T}$ that was moderately relativistic. The thermal parameter value plays a significant role for the stability of our system. Barcons and Lapiedra (1984) [9] gave explicit expressions for the thermodynamic functions of a high-temperature electronpositron plasma and gave expression for distribution functions for a classical dilute arbitrarily hot plasma in equilibrium which we compared our results with it.

Special relativity, however, does not permit velocities greater than the speed of light $c$ and is thus incompatible with a Maxwellian distribution that predicts a non-zero probability for every velocity [10]. It is possible to obtain the relativistic Maxwellian distribution (Jüttner distribution) of a moving gas when the so-called Planck-Einstein case is analyzed. It should be reiterated that the Planck-Einstein theory suffered a modification, principally in the transformation law of energy (the Planck-Einstein case) [11]. Treumann et al. [12] have also shown that the isotropic thermal equilibrium distribution function in relativistic plasma analytically has the form of a modified Jüttner distribution.

A little is known about relativistic distribution functions involving more than two particles, and in particular about the three-particle (or triplet) distribution function. This is of course due to the greater mathematical complexity of higher order correlation functions, and to a lack of a direct link with experiment. Although one can consider experimental determination of triplet distribution function from triple elastic scattering similar to that of the binary distribution function, but to our knowledge, the measurement of three-body correlation function requires the knowledge of the positions of three particles at the same time which is technically very demanding to obtain in 3D samples [13]; this means that the experiment to measure the triplet distribution function directly requires high precision so that the multiple scattering can be differentiated from single scattering. Essentially, one follows the phase-space trajectory of the system as it evolves in time, and has thus the same amount of information as one obtains in a simulation. Similarly the particle positions have measured in dusty plasmas [14]. Lapiedra et al. [15] have undertaken an application of predictive methods to relativistic statistical mechanics. Coulomb forces between point charges are purely repulsive and charges approach very close to each other. Coulomb systems, such as plasma or electrolytes, are made of charged particles interacting through Coulomb's law. The simplest model of a Coulomb system is the one-component plasma (OCP), also called jellium: an assembly of identical point charges, embedded in a neutralizing uniform background of the opposite sign. Here we consider the classical (i.e. non-quantum) equilibrium statistical mechanics of the OCP. It is rather straightforward to calculate higher-order correlation functions from the measured configurations. Moreover we study the model of two-component plasma (TCP) i.e. neutral system of point like particles of positive and negative charges such as electrons and ions. For the numerical calculation we restrict ourselves to the case of (TCP) which anti-symmetric with respect to the charges $e_{e}=-e_{i}=-e$ and therefore symmetrical with respect to the densities $\rho_{e}=\rho_{i}=\rho$. To simplify the numeric investigations, we simulated so far only mass symmetrical electron-ion plasma with $m_{e}=m_{i}=m$.

Homogeneous plasma is characterized by two parameters: the density of particles $\rho$ and the temperature $T$; if there are several kinds of particles, it is also necessary to state their concentrations. There are different energies associated with the plasma, namely:

1) Energy of the rest mass per particle $m c^{2}$;

2) Kinetic energy per particle of order $k T$;

3) Coulomb energy of order $e^{2} \rho^{1 / 3}$ per particle.

The ratios of these energies give us the two main dimensionless parameters of the plasma: the thermal para- 
meter of plasma $\mu=\frac{m c^{2}}{K T}$ and the dilution parameter $\epsilon_{d}=\frac{e^{2} \rho^{1 / 3}}{K T}$. The value of $\mu$ is very important. There are four different regimes characterized by it: plasma with $\mu \leq 1$ is relativistic, $\mu \geq 1$ weakly relativistic plasma, $\mu \gg 1$ low plasma temperature and $\mu \ll 1$ ultra-relativistic high temperatures plasma. In our study we note that the system is safely classical if where $\lambda=\frac{\hbar}{P} \ll \rho^{1 / 3}, \hbar=\frac{h}{2 \pi}, h$ is the Planck constant, and $P$ is the typical linear momentum of particles. A relativistic plasma with a thermal distribution function has temperatures greater than around $260 \mathrm{keV}$, or $3.0 \mathrm{GK}$ (5.5 billion degrees Fahrenheit), where approximately 10\% of the electrons have $\gamma>2$ where $\gamma=1 / \sqrt{1-(v / c)^{2}}$ is the Lorentz factor. It is occurs in many environments in astrophysics, including gamma-ray bursts, AGN jets, and pulsar winds. In physics, a particle is called ultrarelativistic when its speed is very close to the speed of light $c$. Or, similarly, in the limit where the Lorentz factor is very large $\gamma \gg 1$ and $\mu=10^{-2}$. Our study is only valid for dilute plasmas $\left(\epsilon_{d}<1\right)$. The plasma temperatures and velocities also plays a significant role for the stability of our system Figure 1 show qualitative sketch of the four different regimes characterized by the thermal parameter $\mu$ and Lorentz factor $\gamma$. Our model lies in the region of high speeds, $\gamma>2$ and $\mu<1$.

In the astrophysical environment, the fraction of ionized particles varies widely from nearly no ionization in cold regions to fully ionized in regions of high temperature. This leads to a wide range of parameters where astrophysical plasmas can exist. While the astrophysical environment is frequently dominated by the presence of the plasma, this plasma is often strongly influenced by and coupled to the presence of embedded particulates (i.e., dust). These dust grains which range in size from a few nanometers to micron-sized objects can become either positively or negatively charged due to interactions with the background plasma environment and ionizing radiation sources in the astrophysical environment [16]. Understanding the processes that govern these plasma particle interactions is critical to the study of astrophysics. The agglomeration and growth of larger particles from single atoms and dust grains leads to the eventual formation of objects so large that gravity becomes the dominant force controlling their subsequent evolution [17].

Another important point is that of the walls. We shall study plasma which is homogeneous and isotropic but the plasma must be confined or it will expand. We may assume that the plasma is confined by some kind of walls which prevent the escape of particles, but that the container is so large that the effects of the walls are negligible. Our calculations are based on the phase-space distribution function; this is defined as the number of particles per unit volume of space per unit volume of velocity space: At time $t$, number of particles in elementary volume of space, with velocities in range $u \rightarrow u+\mathrm{d} u=f(x, u, t) \mathrm{d}^{3} x \mathrm{~d}^{3} u$.

\section{The Basic Equations and Hierarchy}

The statistical state of a macroscopic system of $N$ particles is in a complete-though in an intractably complex-

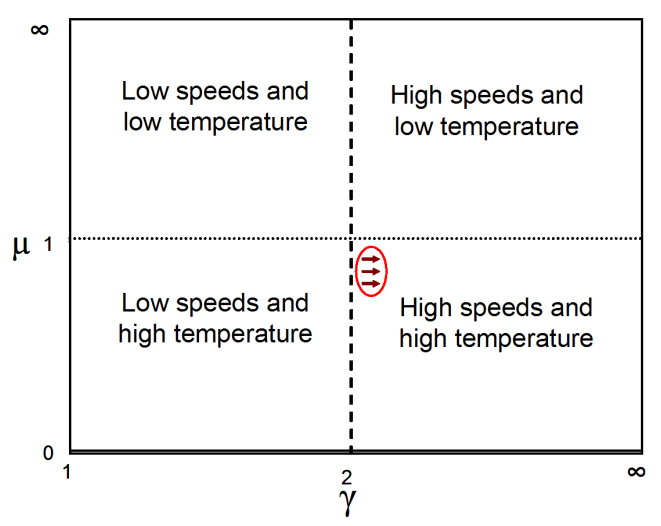

Figure 1. Qualitative sketch of the four different regimes characterized by the thermal parameter $\mu$ and Lorentz factor $\gamma$ : our model lies in the region of high speeds, $\gamma>2$ and $\mu \lesssim 1$. 
way described by the distribution function $F^{N}$ in $6 N$ dimensional phase space, which is spanned by the coordinates and velocities of all individual particles $F^{N}\left(t, x_{A}, u_{A}\right), A=1,2, \cdots, N, x_{A}$ being the three position of particle $A$, and $u_{A}=v_{A} / \sqrt{1-\left(v_{A} / c\right)^{2}}$ the spatial components of its four velocity in a given frame.

The $s$-particle reduced distribution is giving by:

$$
F^{(s)}\left(t, x_{A}, u_{A}\right)=\int F^{N} \prod_{R=s+1}^{N} \mathrm{~d}^{3} x_{R} \mathrm{~d}^{3} u_{R} .
$$

The relativistic BBGKY hierarchy [15] is given by

$$
u_{A}^{\alpha} \frac{\partial F^{(s)}}{\partial x_{A}^{\alpha}}+\sum_{B} \zeta_{A B}^{\alpha} \frac{\partial F^{(s)}}{\partial u_{A}^{\alpha}}+\sum_{R} \int \zeta_{A B}^{\alpha} \frac{\partial F^{(s+1)}}{\partial u_{A}^{\alpha}} \prod_{R=s+1}^{N} \mathrm{~d}^{3} x_{R} \mathrm{~d}^{3} u_{R}=0, \quad A \neq B
$$

where $\zeta_{A B}$ is the acceleration of the charge $A$ in the presence of the charge $B$.

The Einstein summation convention is only valid for Greek labels. Therefore, for each value of $s$ we have $s$ equations, since $A=1,2, \cdots, s$. As in the non-relativistic case, the determination of the reduced generalized distribution function $F^{(s)}$ can only be made when the hierarchy is cut off somewhere, that is, when for some value s we give $F^{(s+1)}$ as a function of the other $F^{(r)}$ functions with $r<s+1$.

According to what we done in non-relativistic case [7] we set, whatever particles 1, 2, 3 are,

$$
\begin{gathered}
F^{(2)}(1,2)=F^{(1)}(1) F^{(1)}(2)\left[1+G(1,2)+\frac{1}{2} G^{2}(1,2)+\frac{1}{3 !} G^{3}(1,2)\right] \\
F^{(3)}(1,2,3)=F^{(1)}(1) F^{(1)}(2) F^{(1)}(3) \\
\times\left[1+G(1,2)+G(1,3)+G(2,3)+\frac{1}{2}(G(1,2)+G(1,3)+G(2,3))^{2}+\frac{1}{3 !}(G(1,2)+G(1,3)+G(2,3))^{3}\right] .
\end{gathered}
$$

Let us consider the case of homogeneous plasma in equilibrium. Then $F^{(1)}$ is the one-particle distribution function of an ideal gas see Figure 2 which shows one particle relativistic distribution function in the particle velocity interval $(0,0.9 c)$ for different values of the thermal parameter $\mu$. That is, in a frame relative to which the system is macroscopically at rest, we must set for $F^{(1)}$ the relativistic Maxwellian distribution [18]

$$
F^{(1)}\left(v_{1}\right)=\frac{\mu_{1}}{4 \pi\left(m c^{3}\right) K_{2}\left(\mu_{1}\right)} \exp \left(\frac{-\mu_{1}}{\sqrt{1-\left(v_{1} / c\right)^{2}}}\right)
$$

where $K_{2}(\mu)$ denotes the modified Bessel function.

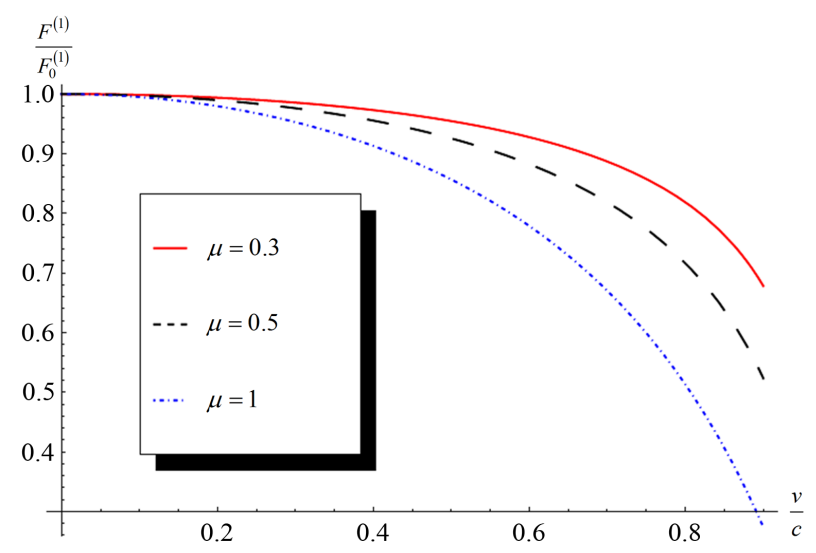

Figure 2. The one particle relativistic distribution function in the particle velocity interval $(0,0.9 c)$ for different values of the thermal parameter $\mu$. 


\section{The Binary Relativistic Distribution Function}

At first sight, the calculation of the relativistic interaction between two charged particles seems rather involved, because the force on particle 1 at time $t$ would depend on the position and velocity of particle 2 at $a$ retarded time. Also, the position and velocity of 1 at that time depends on the position and velocity of 1 at an earlier time, and so on. If one chooses a frame of reference, the acceleration on particle 1 due to the presence of 2 can be calculated from the position and velocity of 2 at that time. This does not mean that actions propagate instantaneously, but rather that there is a precise scheme to take retardation into account automatically, through the equations of the theory. All we shall need in that chapter is the time component of the acceleration of particle 2 due to particle 1 [19].

To calculate the binary relativistic distribution function substituting from Equations (3) and (5) into (2) for $s=1,2$ we obtain

$$
\begin{gathered}
\sum_{R} \int F^{(1)}(R) \zeta_{1 R}^{\alpha} \frac{\partial}{\partial u_{1}^{\alpha}} F^{(1)}(1) G(1, R) \mathrm{d}^{3} x_{R} \mathrm{~d}^{3} u_{R}=0 \\
u_{A}^{\alpha} \frac{\partial G(1,2)}{\partial x_{A}^{\alpha}}-\mu_{A} \zeta_{A B}^{0}+\sum_{R} \int F^{(1)}(R) \zeta_{A R}^{0} G(B, R) \mathrm{d}^{3} x_{R} \mathrm{~d}^{3} u_{R}=0 .
\end{gathered}
$$

Then, by solving the integro-differential Equation (7) and by using the Fourier transform of $G(1,2)=G\left(\boldsymbol{x}, \boldsymbol{v}_{1}, \boldsymbol{v}_{2}\right)$ i.e., $G\left(\boldsymbol{k}, \boldsymbol{v}_{1}, \boldsymbol{v}_{2}\right)=(2 \pi)^{-3 / 2} \int G\left(\boldsymbol{x}, \boldsymbol{v}_{1}, \boldsymbol{v}_{2}\right) \exp (i \boldsymbol{k} \cdot \boldsymbol{x}) \mathrm{d}^{3} x$. We get the relativistic binary generalized distribution function $F^{(2)}(1,2)$ at $t_{1}=t_{2}$ in the following form

$$
\begin{aligned}
& F^{(2)}(1,2)=F^{(1)}(1) F^{(1)}(2) \sum_{i, j=1, i \neq j}^{2}\left\{1-\frac{e_{i} e_{j}}{m_{i} r_{i j}} e^{-\kappa r_{i j}} \mu_{i}+\frac{e_{i} e_{j}}{4 m_{j} \pi^{2}} l_{i j} \mu_{j}+\frac{e_{i}^{2} e_{j}^{2}}{2 m_{i}^{2} r_{i j}^{2}} e^{-2 \kappa r_{i j}} \mu_{i}^{2}+\frac{e_{i}^{2} e_{j}^{2} \mu_{j} \mu_{i}}{2 m_{i} m_{j} \pi^{2} r_{i j}} e^{-\kappa r_{i j}} l_{i j}\right. \\
& +\frac{e_{i}^{2} e_{j}^{2}}{16 m_{j}^{2} \pi^{4}} l_{i j}^{2} \mu_{j}^{2}-\frac{e_{i}^{3} e_{j}^{3}}{6 m_{i}^{3} r_{i j}^{3}} e^{-3 \kappa r_{i j}} \mu_{i}^{3}-\frac{e_{i}^{3} e_{j}^{3}}{64 m_{j}^{3} \pi^{6}} l_{i j}^{3} \mu_{j}^{3}-\frac{3 e_{i}^{3} e_{j}^{3}}{8 m_{i}^{2} m_{j} \pi^{2} r_{i j}^{2}} e^{-2 \kappa r_{i j}} \mu_{i}^{2} \mu_{j} l_{i j} \\
& \left.-\frac{3 e_{i}^{3} e_{j}^{3}}{16 m_{i} m_{j}^{2} \pi^{4} r_{i j}} e^{-\kappa r_{i j}} \mu_{i} \mu_{j}^{2} l_{i j}^{2}+\cdots\right\} \\
& \text { where } \quad \boldsymbol{n}=\frac{\boldsymbol{k}}{k}, \quad \kappa^{2}=\frac{4 \pi}{V} \sum_{R} \frac{\mu_{R}}{m_{R}} e_{R}^{2}, \quad \alpha^{2}=\frac{4 \pi}{V} \sum_{R} e_{R}^{2} \frac{\mu_{R}}{m_{R}} B\left(\mu_{R}\right) \text {, } \\
& l_{i j}=\int \frac{\left(\boldsymbol{n} \cdot \boldsymbol{v}_{i}\right)\left(\boldsymbol{n} \cdot \boldsymbol{v}_{j}\right)-\boldsymbol{v}_{i} \cdot \boldsymbol{v}_{j}}{\left(i+\boldsymbol{n} \cdot \boldsymbol{v}_{i}\right)\left(i+\boldsymbol{n} \cdot \boldsymbol{v}_{j}\right)} \frac{e^{-i \boldsymbol{k} \cdot \boldsymbol{x}}}{k^{2}+\alpha^{2}} \mathrm{~d}^{3} k, \\
& B\left(\mu_{R}\right)=\pi \int F^{(1)}(R)\left[\frac{3-v_{R}^{2}}{2 v_{R}} \ln \frac{1+v_{R}}{1-v_{R}}-3\right] u_{R}^{2} \mathrm{~d} u_{R} .
\end{aligned}
$$

Let us now study the two-particle relativistic distribution for the model of two-component plasma (TCP) i.e. neutral system of point like particles of positive and negative charges such as electrons and ions. For the numerical calculation we restrict ourselves to the case of two-component plasma which anti-symmetric with respect to the charges $e_{e}=-e_{i}=-e$ and therefore symmetrical with respect to the densities $n_{e}=n_{i}=n$.

For two-component plasma we can use the two-particle correlation function $G(1,2)$ which is given by

$$
\begin{gathered}
G(1,2)=g_{1}\left(r_{12}\right)+g_{2}\left(r_{12}\right) \frac{\boldsymbol{v}_{1} \cdot \boldsymbol{v}_{2}}{c^{2}}+g_{3}\left(r_{12}\right) \frac{\left(\boldsymbol{x}_{12} \cdot \boldsymbol{v}_{1}\right)\left(\boldsymbol{x}_{12} \cdot \boldsymbol{v}_{2}\right)}{2 c^{2} r_{12}^{2}} \\
g_{1}\left(r_{12}\right)=-\frac{e_{1} e_{2}}{K T r_{12}} e^{-\kappa r_{12}}
\end{gathered}
$$

where $g_{1}\left(r_{12}\right)$ is the Debye-Hiickel solution [20] for two-component plasma, by substituting Equation (9) into Equation (3) we get the binary distribution function for two-component plasma in the following form: 


$$
\begin{gathered}
F^{(2)}(1,2)=\frac{\mu_{1} \mu_{2}}{8 \pi^{2}\left(m c^{3}\right)^{2} K_{2}\left(\mu_{1}\right) K_{2}\left(\mu_{2}\right)} \sum_{i, j=1, i \neq j}^{2}\left\{1-\frac{e_{i} e_{j}}{m_{i} r_{i j}}\left[e^{-\kappa r_{i j}}+\frac{\boldsymbol{v}_{i} \cdot \boldsymbol{v}_{j}}{2 c^{2}}+\frac{\left(\boldsymbol{x}_{i j} \cdot \boldsymbol{v}_{i}\right)\left(\boldsymbol{x}_{i j} \cdot \boldsymbol{v}_{j}\right)}{2 c^{2} r_{i j}^{2}}\right] \mu_{i}\right. \\
+\frac{e_{i}^{2} e_{j}^{2}}{2 m_{i}^{2} r_{i j}^{2}}+\left[e^{-\kappa r_{i j}}+\frac{\boldsymbol{v}_{i} \cdot \boldsymbol{v}_{j}}{2 c^{2}}+\frac{\left(\boldsymbol{x}_{i j} \cdot \boldsymbol{v}_{i}\right)\left(\boldsymbol{x}_{i j} \cdot \boldsymbol{v}_{j}\right)}{2 c^{2} r_{i j}^{2}}\right]^{2} \mu_{i}^{2} \\
\left.-\frac{e_{i}^{3} e_{j}^{3}}{6 m_{i}^{3} r_{i j}^{3}}\left[e^{-\kappa r_{i j}}+\frac{\boldsymbol{v}_{i} \cdot \boldsymbol{v}_{j}}{2 c^{2}}+\frac{\left(\boldsymbol{x}_{i j} \cdot \boldsymbol{v}_{i}\right)\left(\boldsymbol{x}_{i j} \cdot \boldsymbol{v}_{j}\right)}{2 c^{2} r_{i j}^{2}}\right]^{3} \mu_{i}^{3}+\cdots\right\} \exp \left(\frac{-\mu_{1}}{\sqrt{1-\left(v_{1} / c\right)^{2}}}+\frac{-\mu_{2}}{\sqrt{1-\left(v_{2} / c\right)^{2}}}\right)
\end{gathered}
$$

with $F^{(1)}$ given by Equation (5) (in fact only terms of $F^{(1)}$ up to $1 / c^{2}$ must be retained). It can be verified that $G(1,2)$ given by Equation (9), with $\zeta_{1 R}^{0}$ satisfies identically the condition (6) for $t_{12}=0$, to first order in $1 / c^{2}$.

The standard two-body distribution function for dilute slightly relativistic plasma has been calculated previously by Kosachev and Trubnikov [21] starting from the Darwin Lagrangian. Lapiedra and Santos [19] result agrees with theirs to order $1 / c^{2}$, but not to higher-order terms. We think that these terms are meaningless unless one goes beyond the Darwin Lagrangian, which is only correct to order $1 / c^{2}$. The Lagrangian approach introduces some modification of the expression for the statistical sum and what is more important the use of the restricted Breit-Darwin Hamiltonian leads to the wrong behavior of the pair correlation function and therefore to the incorrect expressions for various thermodynamical quantities [22].

According to our knowledge few scientists studied the binary distribution function for relativistic dilute plasma [15]; the new in our article is using effects of thermal parameter on the values of binary relativistic distribution function for one and two-component plasma.

\section{The Triplet Relativistic Distribution Function}

The triplet distribution function $F^{(3)}(1,2,3)$ is defined in such a way that $F^{(3)}\left(t, x_{i}, u_{i}\right) \mathrm{d} x_{i} \mathrm{~d} u_{i}, i=1,2,3 \quad$ the calculation of the relativistic interaction between three charged particles seems rather involved, because the force on particle 1 at time $t$ would depend on the position and velocity of particles 2 and 3 at a retarded time. Also, the position and velocity of 1 at that time depends on the position and velocity of 1 at an earlier time.

Substituting Equation (4) and (5) into (2) for $s=1,2,3$ we obtain

$$
\begin{aligned}
F^{(3)}(1,2,3)=F^{(1)} & (1) F^{(1)}(2) F^{(1)}(3) \sum_{i, j=1, i \neq j}^{3}\left\{1-\frac{e_{i} e_{j}}{m_{i} r_{i j}} e^{-\kappa r_{i j}} \mu_{i}+\frac{e_{i} e_{j}}{4 m_{j} \pi^{2}} l_{i j} \mu_{j}+\frac{e_{i}^{2} e_{j}^{2}}{2 m_{i}^{2} r_{i j}^{2}} e^{-2 \kappa r_{i j}} \mu_{i}^{2}\right. \\
& +\frac{e_{i}^{2} e_{j}^{2} \mu_{j} \mu_{i}}{2 m_{i} m_{j} \pi^{2} r_{i j}} e^{-\kappa r_{i j}} l_{i j}-\frac{3 e_{i}^{3} e_{j}^{3}}{8 m_{i}^{2} m_{j} \pi^{2} r_{i j}^{2}} e^{-2 \kappa r_{i j}} \mu_{i}^{2} \mu_{j} l_{i j}-\frac{3 e_{i}^{3} e_{j}^{3}}{16 m_{i} m_{j}^{2} \pi^{4} r_{i j}} e^{-\kappa r_{i j}} \mu_{i} \mu_{j}^{2} l_{i j}^{2} \\
& \left.+\frac{e_{i}^{2} e_{j}^{2}}{16 m_{j}^{2} \pi^{4}} l_{i j}^{2} \mu_{j}^{2}-\frac{e_{i}^{3} e_{j}^{3}}{6 m_{i}^{3} r_{i j}^{3}} e^{-3 \kappa r_{i j}} \mu_{i}^{3}-\frac{e_{i}^{3} e_{j}^{3}}{64 m_{j}^{3} \pi^{6}} l_{i j}^{3} \mu_{j}^{3}+\cdots\right\} .
\end{aligned}
$$

If we used the Kirkwood superposition approximation (KSA) [23]; which is consisting of the assumption that the potential in a set of three particles is the sum of the three pair potentials, this is equivalent to assuming that the triplet distribution function is the product of the three radial distribution functions

$$
F^{(3)}(1,2,3)=F^{(2)}(1,2) F^{(2)}(2,3) F^{(2)}(1,3) .
$$

It has been mentioned that before 2003 there is no direct measurement of three-body correlation function and this is the first study for the triplet distribution function in the case of dilute relativistic plasma. Such measurement requires the knowledge of the positions of three particles at the same time which is technically very demanding to obtain in 3D samples [13]; this means that the experiment to measure the triplet distribution function directly requires high precision so that the multiple scattering can be differentiated from single scattering. Now because of the existence of video-microscopy, a modern experimental technique applied to colloidal systems to directly measure all particles' positions at all times. Essentially, one follows the phase-space trajectory of the 
system as it evolves in time, and has thus the same amount of information as one obtains in a simulation. Similarly the particle positions have measured in dusty plasmas [24].

From Equation (12) we can obtain the classical TDF for two-component plasma in the following form:

$$
\begin{aligned}
F^{(3)}(1,2,3)= & F^{(1)}(1) F^{(1)}(2) F^{(1)}(3) \sum_{i, j=1, i \neq j}^{3}\left\{1-\frac{e_{i} e_{j}}{m_{i} r_{i j}}\left[e^{-\kappa r_{i j}}+\frac{\boldsymbol{v}_{i} \cdot \boldsymbol{v}_{j}}{2 c^{2}}+\frac{\left(\boldsymbol{x}_{i j} \cdot \boldsymbol{v}_{i}\right)\left(\boldsymbol{x}_{i j} \cdot \boldsymbol{v}_{j}\right)}{2 c^{2} r_{i j}^{2}}\right] \mu_{i}+\frac{e_{i}^{2} e_{j}^{2}}{2 m_{i}^{2} r_{i j}^{2}}\right. \\
& \left.+\left[e^{-\kappa r_{j j}}+\frac{\boldsymbol{v}_{i} \cdot \boldsymbol{v}_{j}}{2 c^{2}}+\frac{\left(\boldsymbol{x}_{i j} \cdot \boldsymbol{v}_{i}\right)\left(\boldsymbol{x}_{i j} \cdot \boldsymbol{v}_{j}\right)}{2 c^{2} r_{i j}^{2}}\right]^{2} \mu_{i}^{2}-\frac{e_{i}^{3} e_{j}^{3}}{6 m_{i}^{3} r_{i j}^{3}}\left[e^{-\kappa r_{i j}}+\frac{\boldsymbol{v}_{i} \cdot \boldsymbol{v}_{j}}{2 c^{2}}+\frac{\left(\boldsymbol{x}_{i j} \cdot \boldsymbol{v}_{i}\right)\left(\boldsymbol{x}_{i j} \cdot \boldsymbol{v}_{j}\right)}{2 c^{2} r_{i j}^{2}}\right]_{i}^{3}+\cdots\right\} .
\end{aligned}
$$

And we also can used (KSA) which is given in Equation (13) to get it in the form

$$
\begin{aligned}
F^{(3)}(1,3,2)= & \left(F^{(1)}(1) F^{(1)}(2) F^{(1)}(3)\right)^{2}\left\{1-\frac{\mu_{1} e_{1} e_{2}}{m_{1} r_{12}}\left[e^{-\kappa r_{12}}+\frac{\boldsymbol{v}_{1} \cdot \boldsymbol{v}_{2}}{2 c^{2}}+\frac{\left(\boldsymbol{x}_{12} \cdot \boldsymbol{v}_{1}\right)\left(\boldsymbol{x}_{12} \cdot \boldsymbol{v}_{2}\right)}{2 c^{2} r_{12}^{2}}\right]\right. \\
& \left.-\frac{\mu_{2} e_{3} e_{2}}{m_{2} r_{32}}\left[e^{-\kappa r_{32}}+\frac{\boldsymbol{v}_{3} \cdot \boldsymbol{v}_{2}}{2 c^{2}}+\frac{\left(\boldsymbol{x}_{32} \cdot \boldsymbol{v}_{3}\right)\left(\boldsymbol{x}_{32} \cdot \boldsymbol{v}_{2}\right)}{2 c^{2} r_{i j}^{2}}\right]-\frac{\mu_{3} e_{1} e_{3}}{m_{3} r_{13}}\left[e^{-\kappa r_{13}}+\frac{\boldsymbol{v}_{1} \cdot \boldsymbol{v}_{3}}{2 c^{2}}+\frac{\left(\boldsymbol{x}_{13} \cdot \boldsymbol{v}_{1}\right)\left(\boldsymbol{x}_{13} \cdot \boldsymbol{v}_{3}\right)}{2 c^{2} r_{i j}^{2}}\right]+\cdots\right\} .
\end{aligned}
$$

The triplet and quadruple distribution functions as well as binary distribution function must be incorporated for a more accurate and complete discussion of macroscopic equilibrium properties. A little is known about distribution functions involving more than two particles, and in particular about the three-particle (or triplet) distribution functions. This is of course due to the greater mathematical complexity of higher order correlation functions, and to a lack of a direct link with experiment [25]. And this is considered the first study for the triplet distribution functions for dilute relativistic plasma.

\section{Conclusions}

In many physical systems, the description of a plasma as a Coulomb system is sufficient to reproduce most of the properties of interest. If the system is cold enough, the mean velocities of the particles are much smaller than the speed of light, and the charges may be assumed to interact via the instantaneous Coulomb potential. However, at sufficiently high temperatures, this approximation is no longer valid, and the contributions of the relativistic effects (which include, apart from the trivial kinetic corrections and of course all the retardation effects) must be incorporated when studying the equilibrium properties of the system [26].

In the classical (non-quantum) case, the systematic approach adopted here follows the traditional route of the relativistic Bogoliubov-Born-Green-Kirkwood-Yvon (BBGKY) hierarchy for the reduced distribution functions by formal density expansion. Interestingly, this study is the first to display the effect of thermal parameter of plasma in the classical binary and triplet distribution function. Also the triplet relativistic distribution function for dilute plasma was calculated from the relativistic BBGKY hierarchy. We used the results to obtain the analytical forms of the classical triplet distribution functions for one- and two-component plasmas.

In Figure 3 and Figure 4 we noticed that the value of both binary and triplet relativistic distribution function increased when the $\mu$ value decreased at very high temperature. Physically these results seem acceptable because from the definition of the phase-space distribution function which means the number of particles per unit volume of space per unit volume of velocity space, and at high temperature, the velocity of the particles increases and as a result the number of particles increases per unit volume.

Our calculations are grounded in the classical relativistic statistical mechanics. Plasma is non-degenerate. The system is not dense, so one may neglect the contributions of higher order particle interactions. Figure 5 and Figure 6 show that the two- and three-particle distribution functions have become concentrated to ever-smaller region of speed $v$, dramatically increasing the thermal parameter $\mu$. When we made a comparison between the two-particle relativistic distribution function from Equation (11) and the result of Barcons and Lapiedra as shown in Figure 7, the results were nearly similar at very high velocities. 


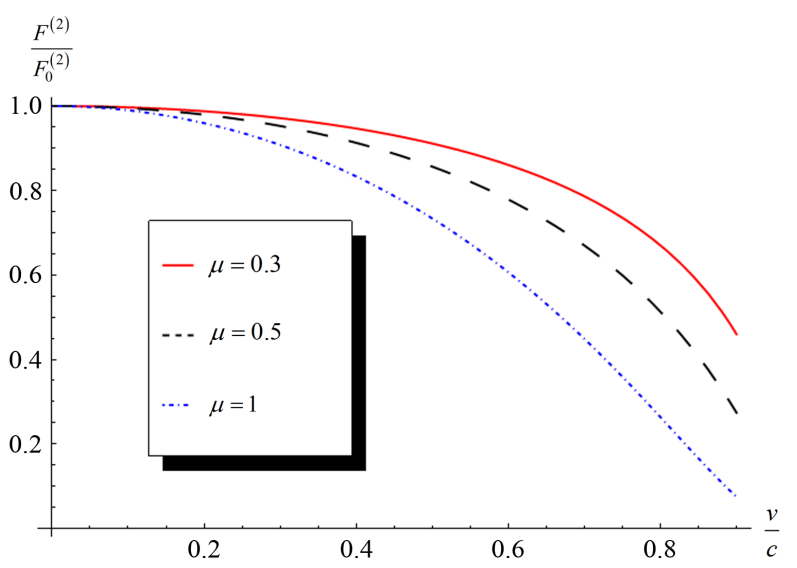

Figure 3. The two-particle relativistic distribution function in the particle velocity interval $(0,0.9 c)$ for one-component plasma.

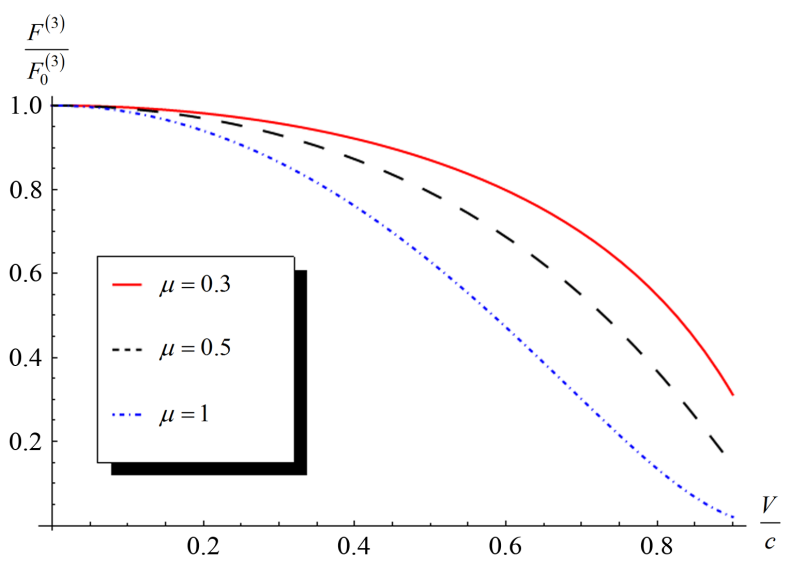

Figure 4. The three-particle relativistic distribution function in the particle velocity interval $(0,0.9 c)$ for one-component plasma for different values of $\mu, v_{1}=v_{2}=v_{3}=v$.

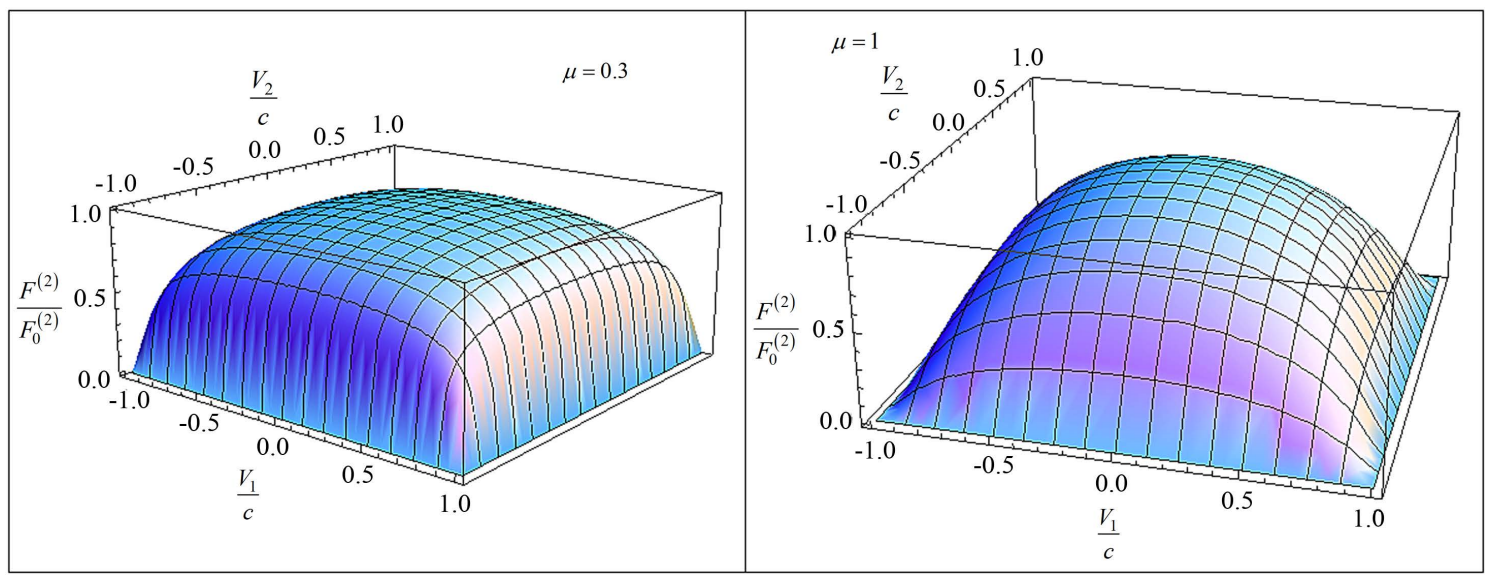

Figure 5. The two-particle relativistic distribution function for two-component plasma in the particle velocity interval $(-c, c)$ at $\mu=1 ; \mu=0.3$ and $v_{1} \neq v_{2}$.

Figure 8 and Figure 9 show that the two- and three-particle distribution functions in the thermal parameter of plasma $\mu$ interval $(0,1)$ for one-component plasma for different values of speed $v=0.8 c, v=0.86 c$, and $v=0.9 c$. We note that the curves are very close to each other, dramatically increasing the thermal parameter 


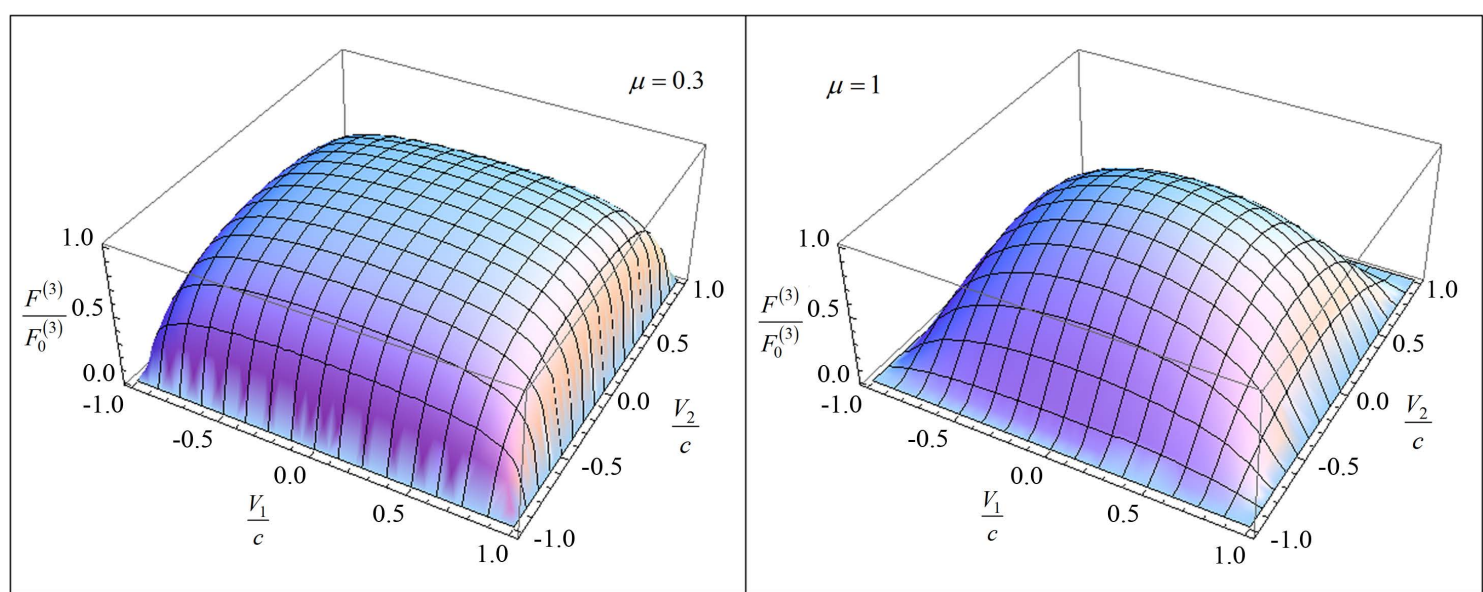

Figure 6. The three-particle relativistic distribution function in the particle velocity interval $(0,0.9 c)$ for one-component plasma for different values of $\mu$; $v_{1}=v_{2}=v_{3}=v$.

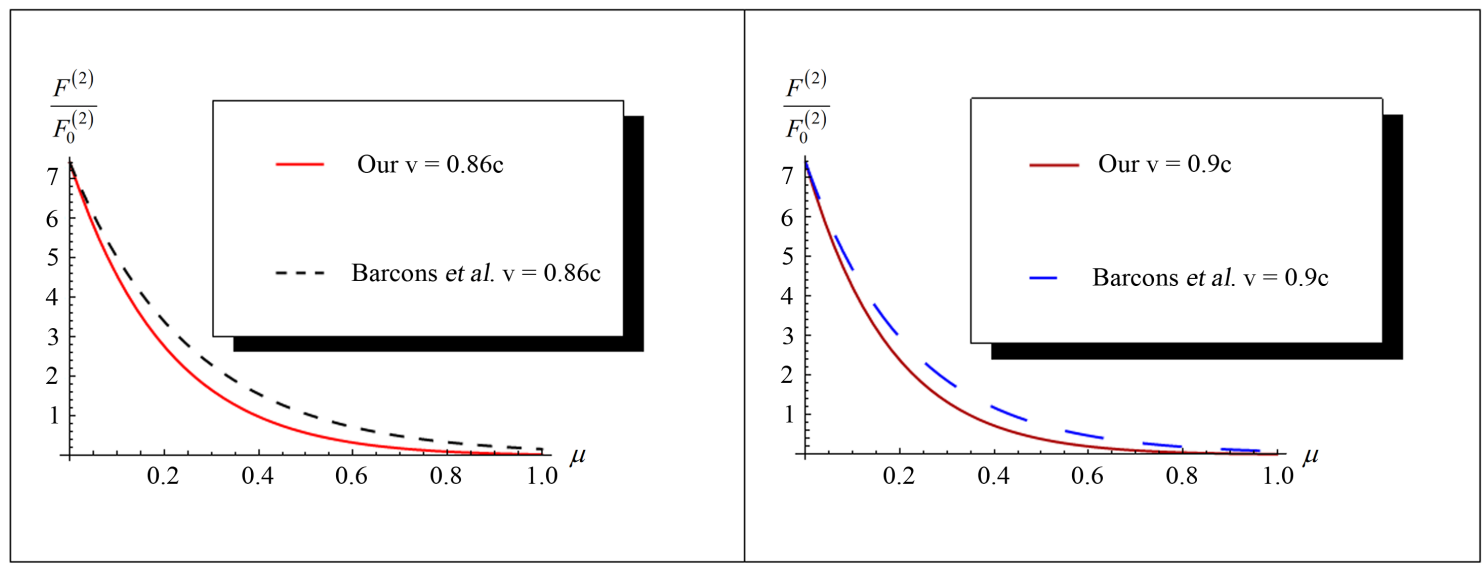

Figure 7. The comparison between $F^{(2)}$ from our result and from Barcons and Lapiedra [9] for $v=0.86 c$ and $v=0.9 c$.

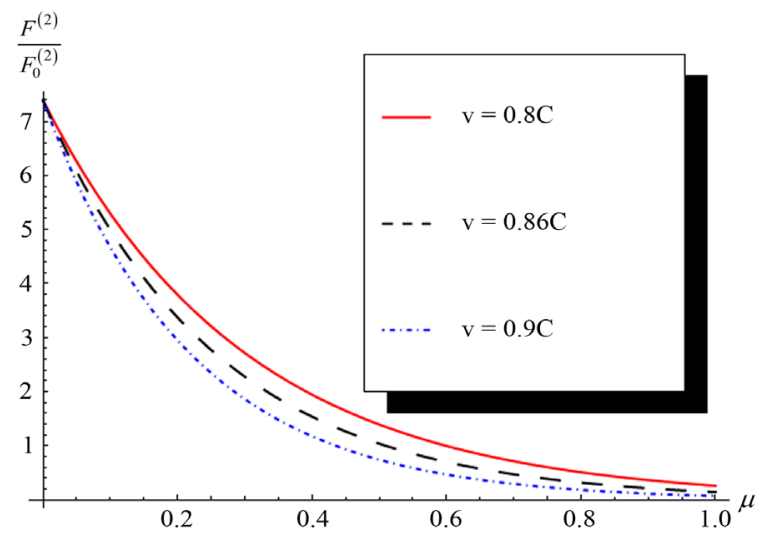

Figure 8. The two-particle relativistic distribution function in the thermal parameter of plasma $\mu$ interval $(0,1)$ for one-component plasma for different values of speed, $v=0.8 c, v=0.86 c, v=0.9 c$.

$\mu$ and for three-particle distribution function more than two-particle distribution function.

Figure 10 shows the comparison between the three-particle relativistic distribution function from Equations (14) and (15). One of them is based on the Kirkwood superposition approximation (KSA) which is consisting of the assumption that the potential in a set of three particles is the sum of the three pair potentials. This is equivalent 


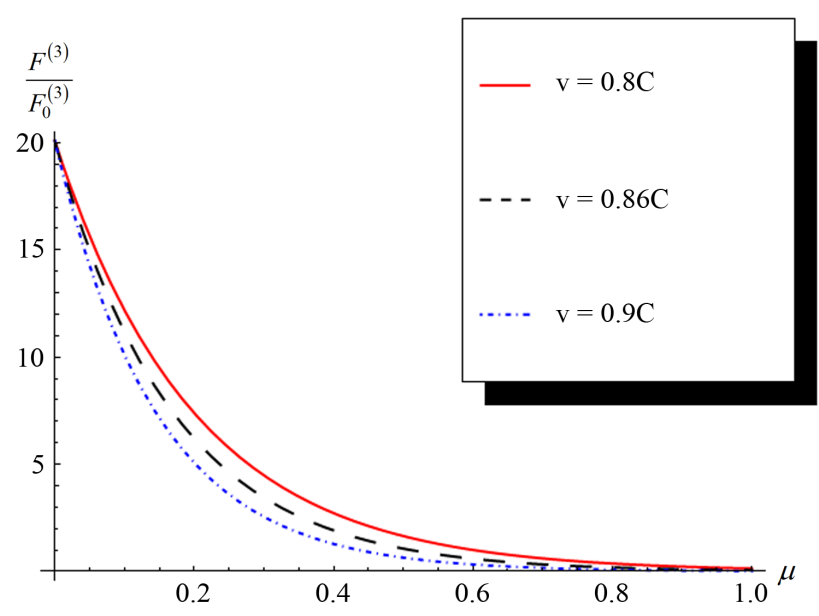

Figure 9. The three-particle relativistic distribution function in the thermal parameter of plasma $\mu$ interval $(0,1)$ for one-component plasma for different values of speed, $v=0.8 c, v=0.86 c, v=0.9 c$.

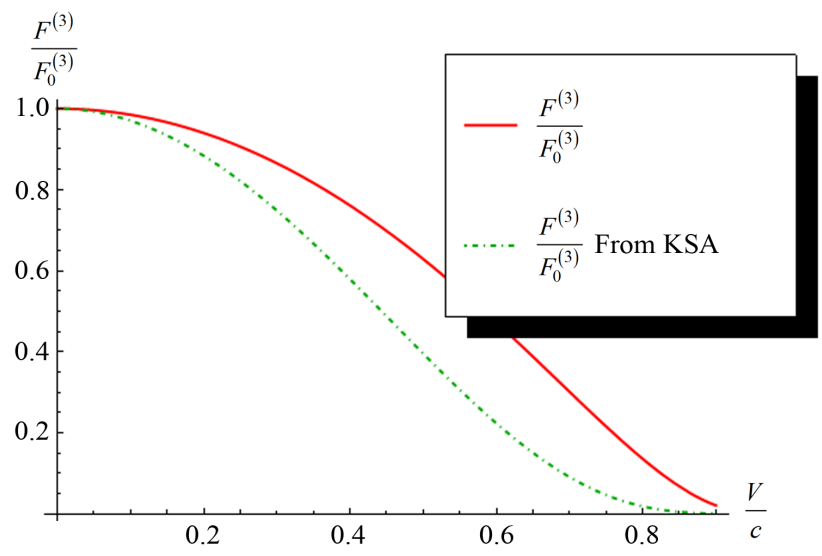

Figure 10. The comparison between $F^{(3)}$ from Equation (14) and $F^{(3)}$ from (KSA) for two-component plasma for $\mu=1$; at $v_{1}=v_{2}=v_{3}=v$.

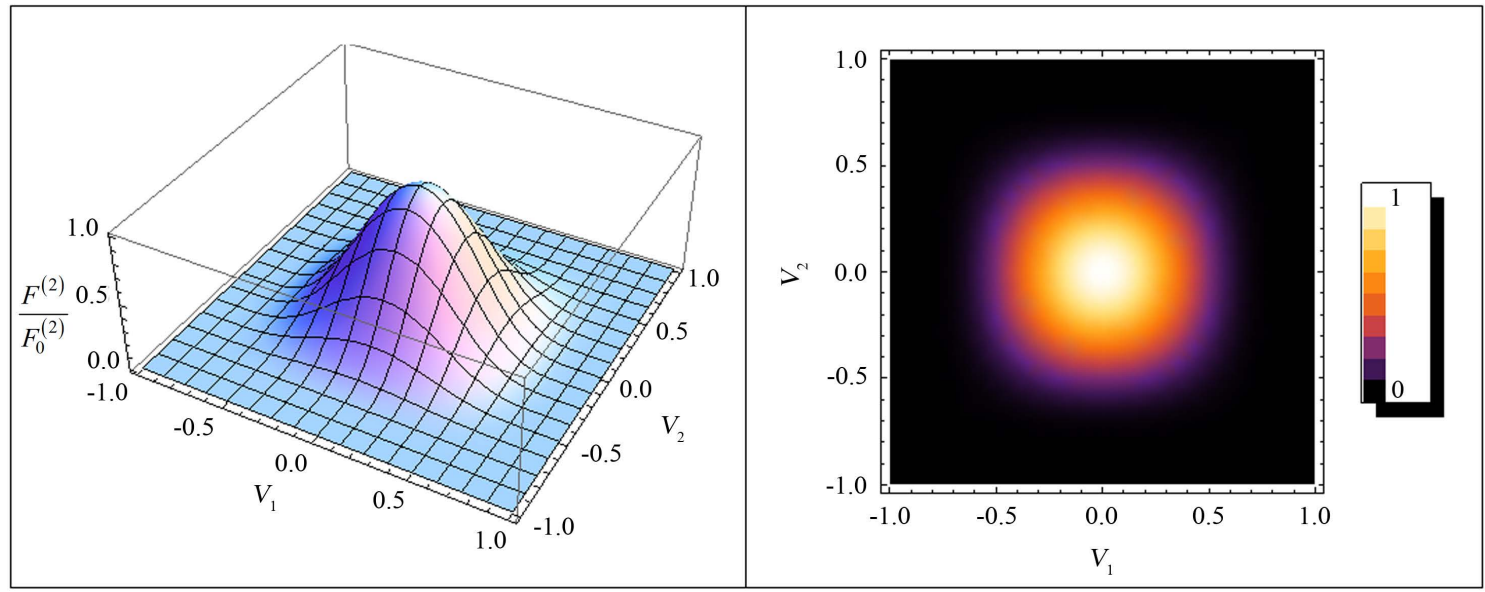

Figure 11. The two-particle relativistic distribution function in the particle velocity interval $(-c, c)$ for $\mu>1$ (weakly relativistic case).

to assume that the triplet distribution function is the product of the three radial distribution functions, and the other form is calculated by using the relativistic BBGKY hierarchy. 


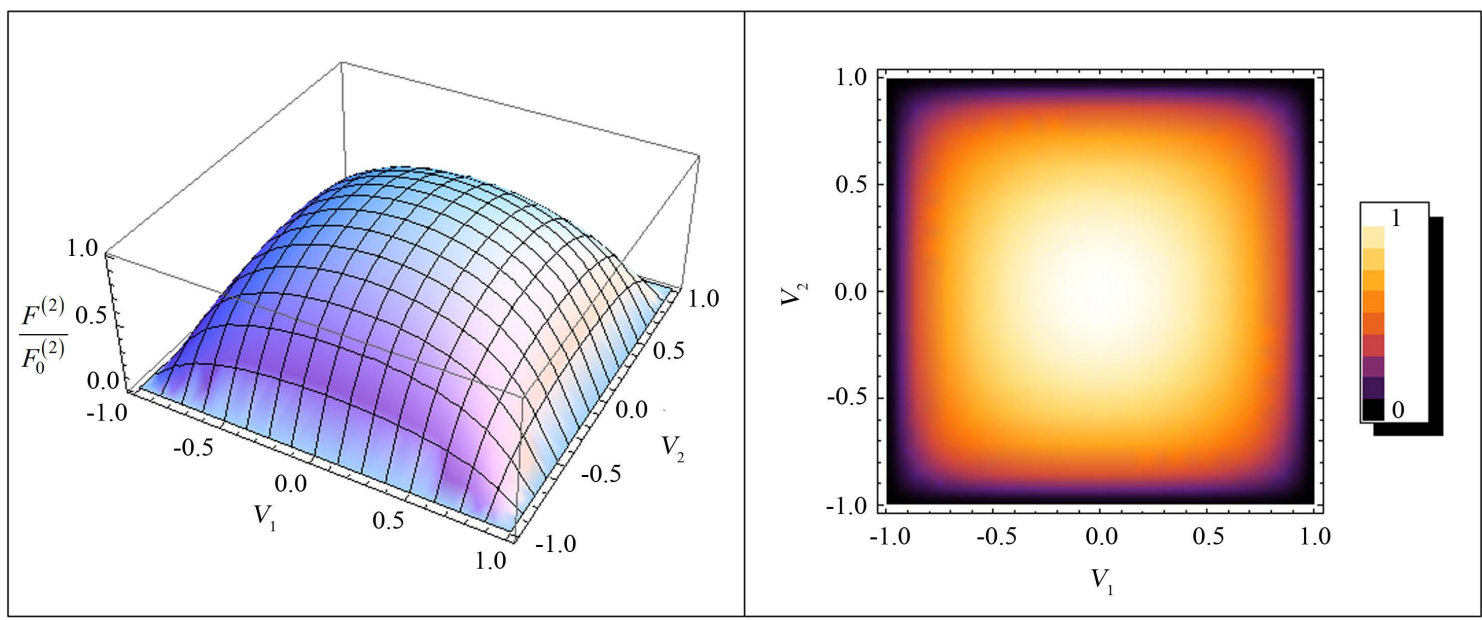

Figure 12. The two-particle relativistic distribution function in the particle velocity interval $(-c, c)$ for $\mu=1$ (relativistic case).

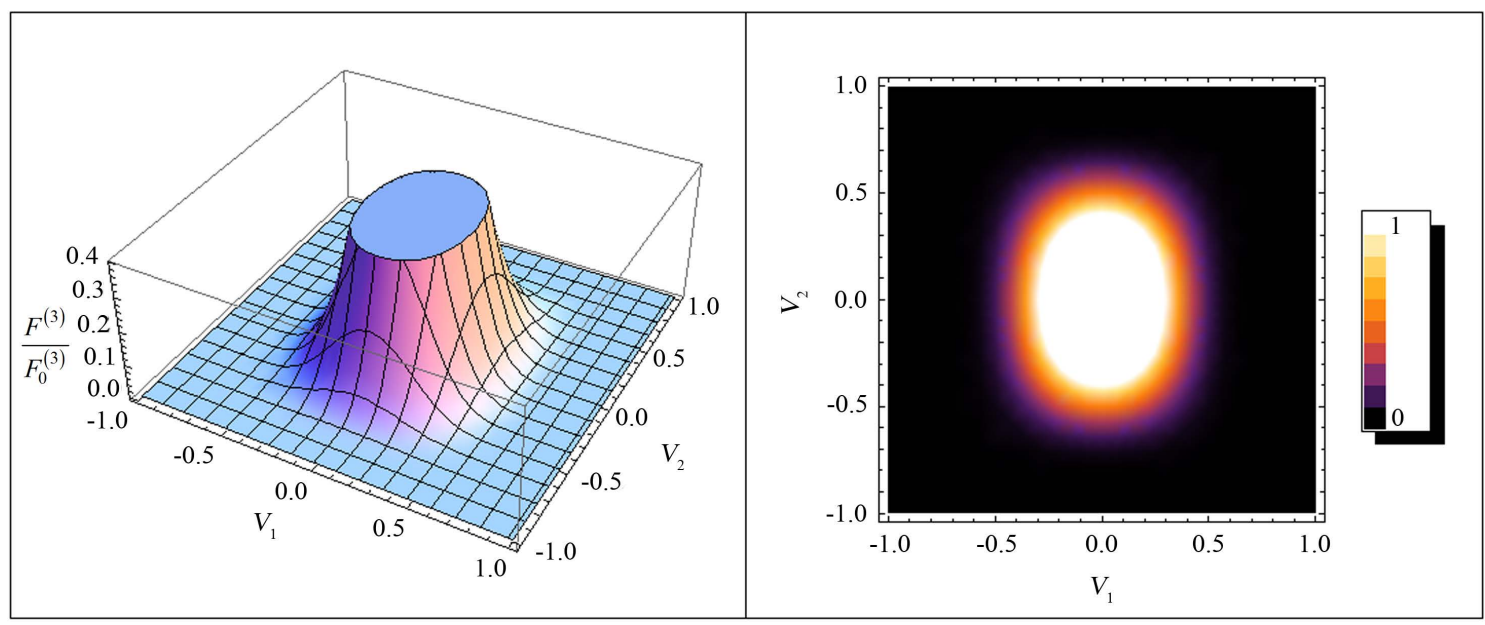

Figure 13. The two-particle relativistic distribution function in the particle velocity interval $(-c, c)$ for $\mu>1$ (weakly relativistic case).

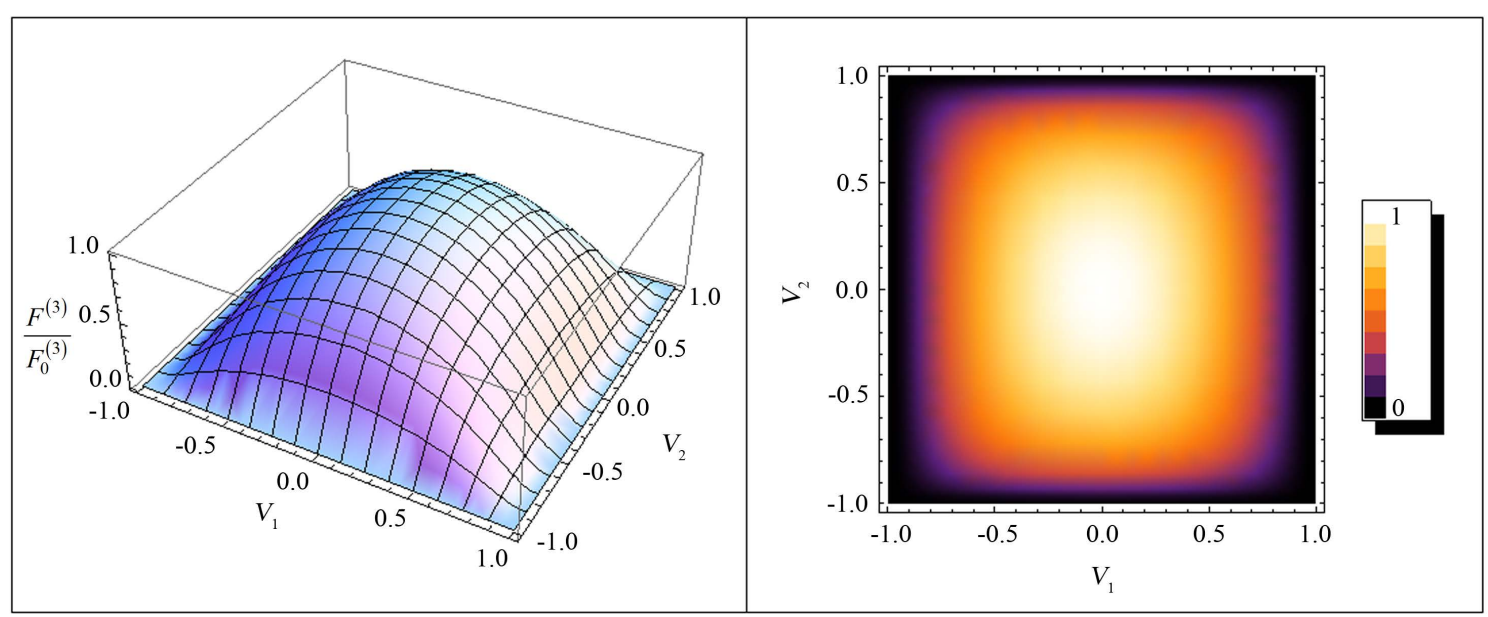

Figure 14. The three-particle relativistic distribution function in the particle velocity interval $(-c, c)$ for $\mu=1$ (relativistic case). 
Finally, we can note that from Figures 11-14, the distribution function has become more concentrated to eversmaller region of speed $v$, dramatically increasing the thermal parameter $\mu$ and for three-particle distribution function more than two-particle distribution function.

\section{References}

[1] Droz-Vincent, P. (1997) Direct Interactions in Relativistic Statistical Mechanics. Foundations of Physics, 27, $363-387$. http://dx.doi.org/10.1007/BF02550162

[2] Bogoliubov, N.N. (1946) Probleme der Dynamischen Theorie in der Statistischen Physik. Moskau.

[3] Simonella, S. (2011) BBGKY Hierarchy for Hard Sphere Systems. Ph.D. Dissertation, Sapienza University of Rome, Rome.

[4] Kraeft, W.D., Kremp, D. and Ebeling, W. (1986) Quantum Statistics of Charged Particle Systems. Akademie Verlag, Berlin. http://dx.doi.org/10.1007/978-1-4613-2159-0

[5] Kahlbaum, T. (1999) Density Expansions of the Reduced Distribution Functions and the Excess Free Energy for Plasma with Coulomb and Short-Range Interactions. Contributions to Plasma Physics, 39, 181-184. http://dx.doi.org/10.1002/ctpp.2150390144

[6] Hussein, N.A. and Eisa, D.A. (2011) The Quantum Equation of State of Fully Ionized Plasmas. Contributions to Plasma Physics, 51, 44-50. http://dx.doi.org/10.1002/ctpp.201110003

[7] Eisa, D.A. (2012) The Classical Binary and Triplet Distribution Functions for Two Component Plasma. Contributions to Plasma Physics, 52, 261-275. http://dx.doi.org/10.1002/ctpp.201100030

[8] Arendt Jr., P.N. and Eilek, J.A. (2000) The Pair Cascade in Strong and Weak Field Pulsars. Pulsar Astronomy-2000 and beyond, 202, 445-448.

[9] Barcons, X. and Lapiedra, R. (1985) Statistical Mechanics of Classical Dilute Relativistic Plasmas in Equilibrium. Journal of Physics A: Mathematical and General, 18, 271-285. http://dx.doi.org/10.1088/0305-4470/18/2/017

[10] Swisdak, M. (2013) The Generation of Random Variates from a Relativistic Maxwellian Distribution. Physics of Plasmas, 20, Article ID: 062110. http://dx.doi.org/10.1063/1.4812459

[11] Ares de Parga, G. and Lopez-Carrera, B. (2011) Relativistic Statistical Mechanics vs. Relativistic Thermodynamics. Entropy, 13, 1664-1693. http://dx.doi.org/10.3390/e13091664

[12] Treumann, R.A., Nakamura, R. and Baumjohann, W. (2011) A Model of So-Called “Zebra” Emissions in Solar Flare Radio Burst Continua. Annales Geophysicae, 29, 1673-1682. http://dx.doi.org/10.5194/angeo-29-1673-2011

[13] Sciortino, F. and Kob, W. (2001) Debye-Waller Factor of Liquid Silica: Theory and Simulation. Physical Review Letters, 86, 648-651. http://dx.doi.org/10.1103/PhysRevLett.86.648

[14] Rosenfeld, Y., Levesque, D. and Weis, J.J. (1990) Free-Energy Model for the Inhomogeneous Hard-Sphere Fluid Mixture: Triplet and Higher-Order Direct Correlation Functions in Dense Fluids. The Journal of Chemical Physics, 92, 6818-6832. http://dx.doi.org/10.1063/1.458268

[15] Lapiedra, R. and Santos, E. (1981) Classical Relativistic Statistical Mechanics: The Case of a Hot Dilute Plasma. Physical Review D, 1, 2181-2188. http://dx.doi.org/10.1103/PhysRevD.23.2181

[16] Alam, M.S., Masud, M.M. and Mamun, A.A. (2014) Effects of Two-Temperature Superthermal Electrons on Dust-IonAcoustic Solitary Waves and Double Layers in Dusty Plasmas. Astrophysics and Space Science, 349, 245-253. http://dx.doi.org/10.1007/s10509-013-1639-3

[17] Masud, M.M., Kundu, N.R. and Mamun, A.A. (2013) Obliquely Propagating Dust-Ion Acoustic Solitary Waves and Their Multidimensional Instabilities in Magnetized Dusty Plasmas with Bi-Maxwellian Electrons. Canadian Journal of Physics, 91, 530-536. http://dx.doi.org/10.1139/cjp-2012-0390

[18] Lazar, M., Stockem, A. and Schlickeiser, R. (2010) Towards a Relativistically Correct Characterization of Counterstreaming Plasmas. I. Distribution Functions. Open Plasma Physics Journal, 3, 138-147. http://dx.doi.org/10.2174/1876534301003010138

[19] Lapiedra, R. and Santos, E. (1983) Classical Dilute Relativistic Plasma in Equilibrium. Two-Particle Distribution Function. Physical Review A, 27, 422-430. http://dx.doi.org/10.1103/PhysRevA.27.422

[20] Turski, L.A. (1974) Pair Correlation Function for a System with Velocity-Dependent Interactions. Journal of Statistical Physics, 11, 1-16. http://dx.doi.org/10.1007/BF01019474

[21] Kosachev, V.V. and Trubnikov, B.A. (1969) Relativistic Corrections to the Distribution Functions of Particles in a High-Temperature Plasma. Nuclear Fusion, 9, 53-56. http://dx.doi.org/10.1088/0029-5515/9/1/006

[22] Bel, L., Salas, A. and Sanchez, J.M. (1973) Approximate Solutions of Predictive Relativistic Mechanics for the Electromagnetic Interaction. Physical Review D, 7, 1099-1106. http://dx.doi.org/10.1103/PhysRevD.7.1099 
[23] Kirkwood, J.G. (1935) Statistical Mechanics of Fluid Mixtures. The Journal of Chemical Physics, 3, 300-313. http://dx.doi.org/10.1063/1.1749657

[24] Bonitz, M., Henning, C. and Block, D. (2010) Complex Plasmas: A Laboratory for Strong Correlations. Reports on Progress in Physics, 73, Article ID: 066501. http://dx.doi.org/10.1088/0034-4885/73/6/066501

[25] Barrat, J.L., Hansen, J.P. and Pastore, G. (1988) On the Equilibrium Structure of Dense Fluids: Triplet Correlations, Integral Equations and Freezing. Molecular Physics, 63, 747-767. http://dx.doi.org/10.1080/00268978800100541

[26] Kalman, G.J., Rommel, J.M., Blagoev, K. and Blagoev, K. (1998) Strongly Coupled Coulomb Systems. Springer, Berlin. http://www.springer.com/physics/particle+and+nuclear+physics/book/978-0-306-46031-9 
Scientific Research Publishing (SCIRP) is one of the largest Open Access journal publishers. It is currently publishing more than 200 open access, online, peer-reviewed journals covering a wide range of academic disciplines. SCIRP serves the worldwide academic communities and contributes to the progress and application of science with its publication.

Other selected journals from SCIRP are listed as below. Submit your manuscript to us via either submit@scirp.org or Online Submission Portal.
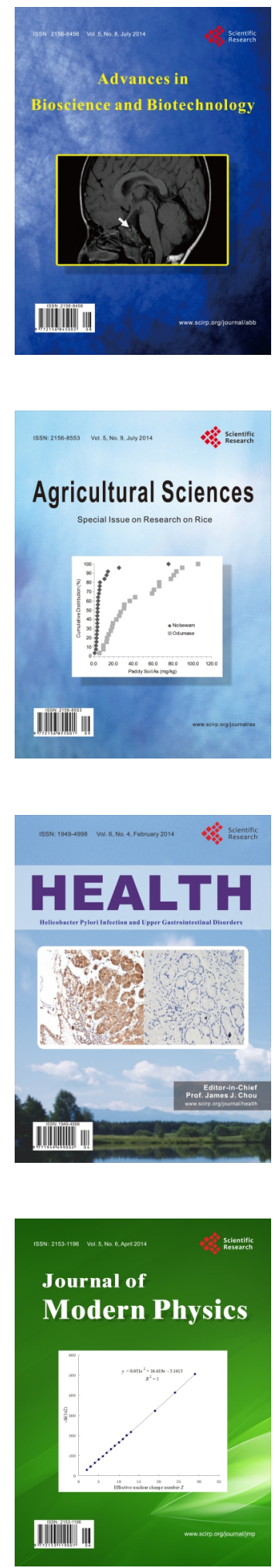
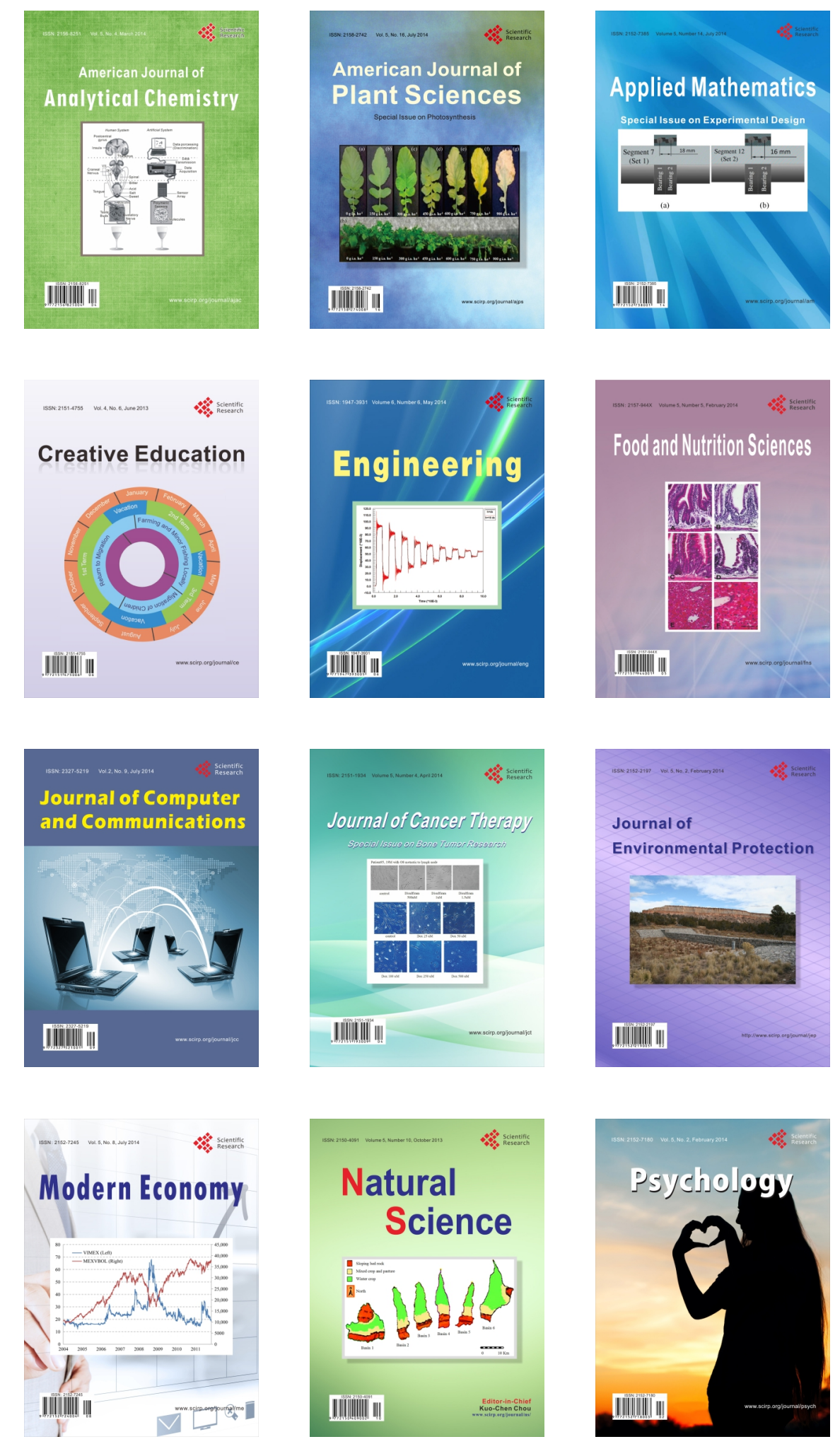